\title{
Observation of an Ultrafast Exciton Transport Regime at Early Times in Quantum Dot Solids
}

\section{Zhilong Zhang}

University of Cambridge https://orcid.org/0000-0001-9903-4945

Jooyoung Sung

University of Cambridge

Daniel Toolan

University of Sheffield

\section{Sanyang Han}

University of Cambridge https://orcid.org/0000-0001-7414-7193

\section{Michael Weir}

University of Nottingham https://orcid.org/0000-0001-8283-1040

\section{James Xiao}

University of Cambridge

\section{Simon Dowland}

University of Cambridge

\section{Mengxia Liu}

University of Cambridge

\section{Anthony Ryan}

University of Sheffield

\section{Richard Jones}

Department of Physics \& Astronomy

\section{Shujuan Huang}

UNSW Australia https://orcid.org/0000-0003-3468-4773

Akshay Rao ( $\square$ ar525@cam.ac.uk)

University of Cambridge https://orcid.org/0000-0003-0320-2962

\section{Research Article}

Keywords: quantum dots, quantum dot solids, ultrafast exciton transport

Posted Date: September 15th, 2020

DOI: https://doi.org/10.21203/rs.3.rs-75982/v1 
License: (c) (i) This work is licensed under a Creative Commons Attribution 4.0 International License. Read Full License

Version of Record: A version of this preprint was published at Nature Materials on March 7th, 2022. See the published version at https://doi.org/10.1038/s41563-022-01204-6. 


\section{Observation of an Ultrafast Exciton Transport Regime at Early}

2

3 Zhilong Zhang ${ }^{1}$, Jooyoung Sung ${ }^{1 *}$, Daniel T. W. Toolan ${ }^{2}$, Sanyang Han ${ }^{1}$, Michael P. Weir ${ }^{3}$,

4 James Xiao ${ }^{1}$, Simon Dowland ${ }^{1}$, Mengxia Liu ${ }^{1}$, Anthony J. Ryan ${ }^{2}$, Richard A. L. Jones ${ }^{3}$,

$5 \quad$ Shujuan Huang ${ }^{4}$ and Akshay Rao ${ }^{1 *}$

$6 \quad{ }^{1}$ Cavendish Laboratory, J.J. Thomson Avenue, University of Cambridge, Cambridge CB3

7 OHE, United Kingdom.

$8 \quad{ }^{2}$ Department of Chemistry, The University of Sheffield, Sheffield S3 7HF, United Kingdom

$9 \quad{ }^{3}$ Department of Physics and Astronomy, The University of Sheffield, Sheffield S3 7RH,

10 United Kingdom

$11{ }^{4}$ Australian Centre for Advanced Photovoltaics (ACAP), School of Photovoltaic and

12 Renewable and Engineering, University of New South Wales, Sydney, 2052 Australia

*email:ar525@cam.ac.uk,js2377@cam.ac.uk 
21 Understanding and engineering exciton transport in quantum dot (QD) solids is both of fundamental interest and crucial to their broad applications in devices ${ }^{1-6}$. Till date, studies of exciton transport in QD solids on pico/nano-second timescales have led to the conclusion that closer packing of QDs enables faster exciton transport, while energetic/structural heterogeneity leads to reduction of exciton diffusivity over time ${ }^{7,8}$. Here we study PbS QD solids using transient absorption microscopy with 13 femtoseconds time resolution and $10 \mathrm{~nm}$ spatial precision. We find exciton diffusivities in the range of $\sim 10^{2} \mathrm{~cm}^{2} \mathrm{~s}^{-1}$ within the first few hundred femtoseconds after photoexcitation, followed by the transition to a slower transport regime with diffusivities in the range $10^{-}$

1 to $1 \mathrm{~cm}^{2} \mathrm{~s}^{-1}$. Counterintuitively, the initial diffusivity is higher and the time before the transition to the slower transport phase is longer in QD solids with longer ligand lengths. This suggests a transition from early time transport of delocalized excitons to later time heterogeneity accelerate the localization process. Our results reveal a new regime for exciton transport in QD solids and provide design rules to engineer desired transport properties in these systems on a range of timescales.

Colloidal QDs have attracted great interest for fundamental studies of exciton and charge dynamics in semiconductor nanostructures, as well as for their applications in various devices including photodetectors ${ }^{3}$, light emitting diodes (LEDs) ${ }^{4}$, photovoltaics ${ }^{5,6}$ and field effect transistors (FETs $)^{9}$. One of the fundamental physical processes underlying the function of these devices is exciton transport in the QD active layer, which is believed to be determined via the assembly of QDs within the film ${ }^{1-4,10,11}$. The requirement for exciton transport however differs between different applications, for example, a photovoltaic cell requires fast exciton transport to the charge-separating interfaces ${ }^{5,6}$, whereas in a LED this can lead to quenching of 
46 photoluminescence ${ }^{1,4}$. A comprehensive understanding of exciton transport physics in QD

47 solids is therefore needed, with the eventual aim of controlling these transport properties in 48 order to optimize device performance.

50 In comparison to the parallel issue of charge transport, the understanding of exciton transport 51 in QD solids is less developed. This partly arises due to limitations of experimental techniques.

52 While FETs and Hall measurements under steady-state conditions provide a wealth of 53 information on charge transport ${ }^{1,2,9}$, excitons cannot be easily probed electrically in ensemble 54 measurements. Coupled with their short-lifetime ( $<5 \mu$ s normally) and short diffusion lengths 55 (in comparison to charges), this limits studies of exciton transport to quenching or optical 56 microscopy measurements. Time-resolved microscopy has been particularly powerful in 57 probing exciton transport, especially at linking transport to underlying disorder in the materials 58 and probing time-dependent diffusion of excitons ${ }^{7,8,12-14}$. The general consensus that emerges 59 from these studies is that closer packing of QDs enables faster exciton transport, while 60 energetic/structural heterogeneity leads to reduction of exciton diffusivity over time ${ }^{7,8}$. These 61 conclusions together with the extracted diffusivities (ranging from $10^{-3}$ to $10^{-2} \mathrm{~cm}^{2} \mathrm{~s}^{-1}$ ) fit well 62 within the picture of Förster resonance energy transfer (FRET) dominated hopping of excitons 63 within a disordered energy landscape ${ }^{14}$. However, due to experimental limitations these studies 64 have probed exciton transport in QD solids on pico/nano-second timescales following 65 photoexcitation. As exciton behaviour can be time-dependent, those conclusions can also be 66 limited by the timescale measured. One important part of the picture is therefore still missing 67 - exciton transport at early times (femtosecond timescale) following photoexcitation. 
69 Here we probe this unexplored regime of exciton transport in QD solids by directly imaging

70 exciton motion on femtosecond (fs) timescale following generation. Surprisingly, we 71 discovered that excitons first undergo very fast transport (diffusivity range of $\sim 10^{2} \mathrm{~cm}^{2} \mathrm{~s}^{-1}$ )

72 within $\sim 300$ fs after photoexcitation and then switch into a much slower transport regime $\left(\sim 10^{-}\right.$

$73{ }^{1}$ to $1 \mathrm{~cm}^{2} \mathrm{~s}^{-1}$ ). Intriguingly, reducing the interdot distance in the QD solids only enhances

74 transport in the slower regime, while it unexpectedly diminishes the initial fast regime. We 75 suggest that both QD packing density and heterogeneity have significant impacts on these 76 transport regimes and the transition between them.

To directly monitor exciton transport at early times, we performed femtosecond transient absorption microscopy (fs-TAM) measurement on the QD films (Fig. 1a). A full description of the instrument is provided in Supplementary Methods. Briefly, a near diffraction-limited pump beam (an effective width $\sigma$ of $107 \pm 7.5 \mathrm{~nm}$ ) with transform-limited pulse duration $(9.2 \mathrm{fs})$ is delivered onto the QD film, generating a Gaussian-shaped exciton density instantaneously. The spatial evolution of exciton distribution is recorded by imaging it with a loosely focused (a width of $6.4 \mu \mathrm{m})$ and transform-limited (6.8 fs) probe pulse at a series of variable time delays between pump and probe pulses. It should be noted that although spatial resolution is still limited by diffraction, by comparing subsequent TAM images it is possible to distinguish the changes in the shape of the distribution as small as $10 \mathrm{~nm}$ (Supplementary Methods) ${ }^{15,16}$. Thus the fs-TAM methodology allows us to study exciton motion with at least 13 fs temporal resolution and $10 \mathrm{~nm}$ spatial precision.

91 Lead sulfide (PbS) QDs (bandgap of $1.68 \mathrm{eV}$ as-synthesized) were chosen as the material 92 platform in this work, as they are among the most intensively studied materials for QD-based 
93 devices due to the highly tuneable band gap, relatively good stability, carrier transport and

94 photoluminescence ${ }^{2,4,5}$. A series of $\mathrm{PbS}$ QD solid-state films with systematic variation in the interdot spacing was prepared. This was achieved by solution-phase pre-exchange of the native oleic acid (OA) capping ligands arising from the synthesis to those with shorter carbon chains

97 (Fig. 1b), namely dodecanoic acid (12C), octanoic acid (8C), hexanoic acid (6C) and butyric acid (4C) before film fabrication by spin-coating (Supplementary Methods). Hereafter, the QD

99 films are denoted as the corresponding ligands. The samples were encapsulated prior to optical characterizations. Both the PL and excitonic features of the absorption spectra were maintained, indicating successful ligand exchange without noticeable aggregation (Supplementary Figs. 1$3)$.

Conventional ensemble level transient absorption (TA) features of the QD films were explored before the fs-TAM measurements (Supplementary Figs. 4-8). As shown in Fig. 1c, gradual redshifts of the photo-bleaching peaks were observed (from $730 \mathrm{~nm}$ of OA) in the TA spectra of the films with reducing ligand lengths, which is consistent with shorter interdot distance and stronger coupling ${ }^{7,17}$. It is noticeable that there was little signal decay within the measurement time window of fs-TAM (kinetics in Supplementary Fig. 9), suggesting very little recombination on these early timescales and hence this is unlikely to affect the transport measurements.

The fs-TAM images of each film were recorded at the probe wavelength corresponding to the photo-bleaching peaks (with a bandwidth of $10 \mathrm{~nm}$, shaded areas shown in Figure 1c). Representative fs-TAM images obtained from OA at early time are shown in Fig. 1d, while data sets for the other samples can be found in Supplementary Figs. 10-13. The series of fs- 
117 TAM images clearly show spatial expansion of exciton distribution in the QD film. Since the 118 initially generated exciton distribution resembles the diffraction-limit pump beam and the 119 spatial expansion occurs isotropically, we fitted the fs-TAM images with an isotropic twodimensional Gaussian function and extracted the corresponding width, $\sigma$, as shown in Fig. 1e (and Supplementary Fig. 14). Near time-zero, the initially created exciton distribution gives a width of $172 \pm 8 \mathrm{~nm}$, which is similar to the distribution width $(180 \mathrm{~nm})$ calculated by convoluting diffraction-limited pump and probe beams ${ }^{15}$. By 260 fs this distribution expands to $224 \pm 8 \mathrm{~nm}$, clearly indicating exciton transport at early times in the QD film.

To quantitatively compare the transport dynamics in QD films, we employ the widely adopted approach of quantifying the spread of spatial exciton distribution by monitoring the change in profile variance, i.e. the mean squared displacement $\left(\mathrm{MSD}=\sigma_{t}^{2}-\sigma_{0}^{2}\right) \operatorname{model}^{7,12,15,16}$. For quantitative comparison, the MSD profiles of the films over time are plotted in Fig. 2a. Interestingly, two distinct transport regimes seem to exist in the MSD profiles: a very fast initial expansion of the exciton distribution within the first $\sim 300$ fs upon photoexcitation, followed by a much slower expansion (up to 4 ps time window of measurement). To test whether it is a two-step diffusion process or an anomalous diffusion process, we fitted the MSD evolution with the power law equation as $\mathrm{MSD}=2 D t^{\alpha}$, where $D$ is the diffusivity and $\alpha$ is the diffusion exponent ${ }^{7,14,15,18}$. We noticed that the MSD profiles cannot be fitted by a single power law equation, due to the abrupt transition from the fast to the slow expansion stage (Supplementary Discussion 1 and Supplementary Fig. 15). Instead, the profiles can be well-fitted within separated time ranges for all samples. The fitting results of $\mathrm{OA}$ is shown in Fig. $2 \mathrm{~b}$ as an example. Within the range of $t_{0}<t<t_{\text {fast }}$, the dynamics can be well described by a power law equation where $\alpha=1$, signifying that the initial exciton transport is diffusive $e^{7,8,14,15,18}$. The corresponding diffusivity $\left(D_{\text {fast }}\right)$ can therefore be extracted from the slope of the fitted line. 
142 Similarly, for $t>t_{\text {slow }}$ the MSD profile can also be described by diffusive motion $(\alpha=1)$ with 143 a much smaller diffusivity $\left(D_{\text {slow }}\right)$. A transition stage (where $t_{\text {fast }}<t<t_{\text {slow }}$ ) also exists between 144 the fast and slow diffusive stages. Alternatively, the slow diffusive region can be described by 145 a subdiffusive motion where $\alpha<1$ (Supplementary Table. 2) by including the transition stage 146 (blue dotted curve, Fig. 2b). We note here that whether we take the description of diffusive or 147 subdiffusive motion for the later slow transport the conclusion is consistent with that discussed 148 below (Supplementary Discussion 2).

Interestingly, the extracted diffusivities from the fast and slow transport stages have opposite response to the reduction of ligand length in the series of QD films (Fig. 2c). It has been generally believed that reducing the ligand length can lead to shorter interdot distance, hence improved QD coupling and transport ${ }^{1-3}$. Consistent with this general perception, the obtained diffusivities in the slow regime increases with shorter ligands in the QD films ( $D_{\text {slow }}$, red dots, Fig. 2c), apart from 4C. The values of $D_{\text {slow }}$ from 0.6 to $1.5 \mathrm{~cm}^{2} \mathrm{~s}^{-1}$ were also comparable with exciton diffusivities reported in other QD systems ${ }^{7,8}$, suggesting the slow stage is likely governed by classical FRET hopping regime ${ }^{14}$.

Intriguingly, the extracted diffusivities from the fast region $\left(D_{\text {fast }}\right)$ decrease with reducing ligand length, opposite to general perception of QD solids and the slow transport regime. The highest $D_{\text {fast }}$ was obtained from OA (with the longest ligand), followed by $12 \mathrm{C}, 8 \mathrm{C}$ and $6 \mathrm{C}$ 162 (black dots, Fig. 2c). 4C was again an exception, while its $D_{\text {fast }}$ was higher than those with the medium-length ligands, however still lower than that of OA. Moreover, the values of the extracted $D_{\text {fast }}$ are within the range from 95 to $280 \mathrm{~cm}^{2} \mathrm{~s}^{-1}$, which is three to four orders of magnitude higher than those from $D_{\text {slow }}$. We emphasize that such an early-time process of 
exciton transport in QD solids is revealed here experimentally for the first time. It has been well demonstrated that the exciton diffusivity of disordered systems such as QD and organic semiconductor solids decrease over time $e^{7,12,13,18}$. Therefore higher diffusivities in such an earlytime range $(<300 \mathrm{fs})$ can be expected. Nevertheless, the observed $D_{\text {fast }}$ values are obviously too high to be described by classical hopping in QD solids, implying the fast regime is likely governed by a different mechanism. Interestingly, the estimated $t_{\text {fast }}$ also shows the same behaviour as $D_{\text {fast }}$ (black dots, Fig. 2d), indicating that films with longer ligands can sustain the initial fast transport for longer time. Similarly, the trend in $t_{\text {slow }}$ also suggests longer ligands postpone the transition from the fast to the slow stage (red dots, Fig. 2d).

Important questions then arise as to what are the underlying mechanisms that dictate the two distinct transport regimes and why they have reversed trends of diffusivities when the ligand length was reduced. To further elucidate these, we looked into the quality and actual packing of the QD solids with different ligands. We first compared the PLQY of the QD solids with those of the corresponding QD solutions (Fig. 3a). The PLQY of the QDs in solution were all around $35 \sim 40 \%$, indicating they had similar level of surface defects and aggregation. In contrast, the drop of PLQY when cast into films was more significant with shorter ligands, consistent with the expectation of better QD coupling and concomitant PL quenching ${ }^{1,19}$. We then performed grazing incidence small angle x-ray scattering (GISAXS) to reveal the actual interdot distance and packing heterogeneity (Fig. 3b and Supplementary Fig. 16). We found the average interdot distance (core-to-core) decreased from $\sim 37.2 \AA$ for OA to $\sim 31.5 \AA$ for both $8 \mathrm{C}$ and $6 \mathrm{C}$, whilst $4 \mathrm{C}$ maintained an unexpectedly large separation of $\sim 35.5 \AA$ (red dots, Fig. 3c). The QD packing disorder (FWHM of the peaks) however gradually increased with shorter ligands (black dots, Fig. 3c). We therefore attribute the unexpectedly large interdot distance of 4C to its high packing heterogeneity (Supplementary Discussion 3), which may have also 
caused $4 \mathrm{C}$ to be an exception to the trend of diffusivities from Fig. 2c. We then correlate the diffusivities from Fig. 2c with the actual interdot distance of the films, and again confirm that $D_{\text {slow }}$ increases with reduction of the actual interdot distance, while $D_{\text {fast }}$ has the opposite trend (Fig. 3d).

Both the trend and values of $D_{\text {slow }}$ suggest that the slow regime is governed by a classical hopping mechanism of localized excitons. In contrast, the high values of $D_{\text {fast }}$ and its trend with interdot distance are highly unusual. This phase of transport is still diffusive in nature (as $\alpha=$ 1), which would seem to rule out ballistic or coherent exciton motion ${ }^{14,20,21}$. One possible explanation is the transport of delocalized rather than localized excitons at early times ${ }^{10,22}$ (also see Supplementary Discussion 4). Theoretical prediction suggests that delocalized excitons can transfer to sites that are spatially far away due to the enhanced net transition dipole moment from superposition of individual QD wavefunctions, leading to much higher diffusivity, via 'supertransfer' mechanism ${ }^{23,24}$. Further experimental and theoretical investigations are called for to investigate this phenomena at early times.

The transition from the fast to the much slower transport regime observed from our results can then be explained as the switch to the diffusion of localized excitons from the early time delocalized transport. We note the transition time between the two regimes is shorter in QD films with higher level of packing disorder $\left(t_{\text {trans }}=t_{\text {slow }}-t_{\text {fast }}\right.$, Fig. 3e), although it is difficult to decouple this from the effect of shorter interdot distance. Nevertheless, despite the fact that $4 \mathrm{C}$ has an average interdot distance between those of $\mathrm{OA}$ and $8 \mathrm{C} / 6 \mathrm{C}$, it has the shortest $t_{\text {trans }}$ among all (Fig. 3e), clearly suggesting packing disorder accelerates the transition from the fast to the slow transport regime. 
216 The important remaining question is why the initial fast transport is less efficient in QD solids

217 when decreasing interdot distance (Fig. 3d). Here, we suggest that one aspect of disorder that

218 has been largely overlooked may cause this - the QD packing density that increases with

219 reduction of interdot distance (Supplementary Fig. 17). Conventional sources of disorder in

220 QD solids include size polydispersity, surface defect states and packing heterogeneity ${ }^{5,7,9}$. But

221 assuming the same polydispersity and defect level per QD, increasing the packing density

222 would unavoidably increase the number of QDs contributing to disorder within the same

223 volume. This would introduce more scattering sites into the transport path of a delocalized

224 exciton initially generated and accelerate the transition to the localized transport regime.

225 Similar observations have been made in carrier transport of organic semiconductors such as

226 rubrene thin films, where the highest mobility was found in a packing direction with larger

227 intermolecular distance ${ }^{25,26}$. We speculate that this may be origin of the less efficient fast

228 transport of delocalized excitons and rapid transition to slow regime in QD solids with shorter

229 interdot distance.

With the above results, we depict the exciton transport profile in the early timescale as shown in Fig. 4. Immediately after photoexcitation, delocalized excitons are generated in the QD solids. They then diffuse quickly in the solid (fast transport regime), and during this stage when an exciton encounters a shallow trap state it may even have sufficient energy to escape as it is still undergoing a cooling process (as shown by TA kinetics in Supplementary Fig. 9) ${ }^{15}$. In the case of a QD solid with larger (but still closely-packed) interdot distance and/or homogeneous packing (Fig. 4a), a low level of disorder allows the fast transport to be faster and sustained for longer. However, when an exciton is eventually localized, the transport mechanism switches 
239 to a hopping (slow) regime that is much less efficient with a longer QD separation. In the case

240 of a QD solid with shorter interdot distance (Fig. 4b), the higher packing density can introduce

241 more QDs as the sources of disorder/scattering sites for a delocalized exciton. A faster

242 localization process occurs and limits the fast transport rate and duration. High packing

243 heterogeneity can further accelerate the localization process. Once the exciton is localized,

244 however it can still transport through the QDs relatively efficient by hopping due to the short

245 interdot separation, as one would normally expect in longer timescales.

247 In summary, our work has revealed the early time exciton dynamics in QD solids. We observed

248 the transition from a very fast to a slow regime over time, which we suggest to be related to the 249 localization process of delocalized excitons. While the slow regime was comparable with 250 classical hopping, the fast regime demonstrated diffusivities of three to four orders of 251 magnitudes faster than those of the slow regime, as well as other reports on exciton transport 252 on longer timescales ${ }^{7,8}$. Counterintuitively, the early-time regime was faster and sustained for 253 longer in QD solids with relatively larger interdot distance, opposite to the classical longer254 time hopping transport process. We attribute this to disorders arising from higher QD packing 255 density (hence disorder density) and structural heterogeneity that accelerate exciton 256 localization. This study sheds light on the factors that dictate exciton transport in QD solids on 257 fs timescales and provides design rules to engineer QD solids to tailor the desired transport 258 properties in these systems on a range of timescales. 

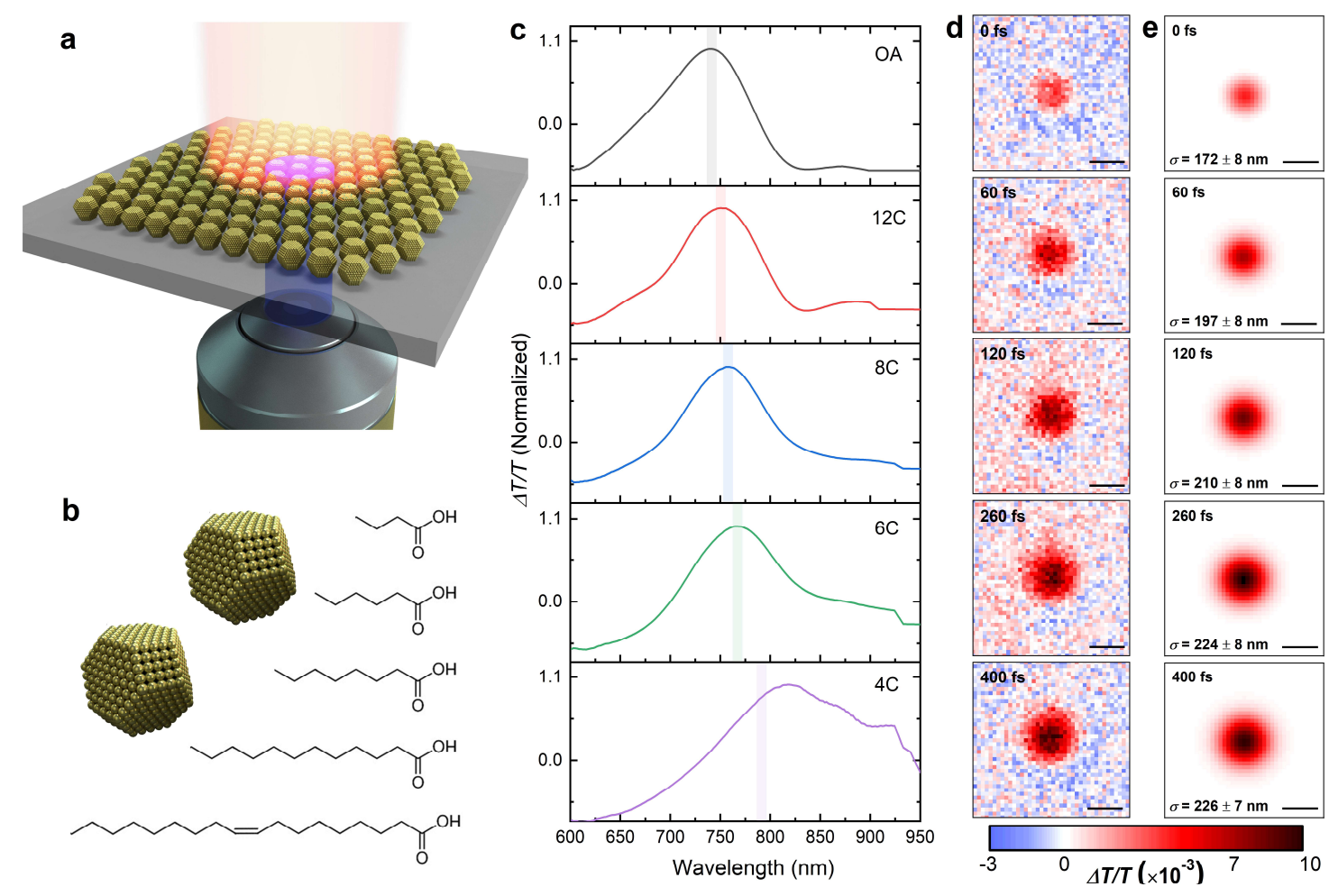

Fig. 1 The fs-TAM measurement of QD thin films with different capping ligands as

discussed in this work. a, Schematic representation of the fs-TAM measurement. A near

diffraction-limited ( $\sigma$ of $107 \pm 7.5 \mathrm{~nm})$ and transform-limited $(9.2 \mathrm{fs}, 580 \mathrm{~nm})$ pump beam is

delivered onto the QD film (blue beam), together with a counter-propagating loosely focused ( $\sigma$ of $6.4 \mu \mathrm{m})$ and transform-limited (6.8 fs) probe pulse (orange beam). Note that the actual samples are thicker films containing multiple layers of QDs with total thickness about 200 $\mathrm{nm}$. b, The interdot distance of the films were modified by the selection of surface ligands. The ligand exchange was performed to replace the original $\mathrm{OA}$ ligands to $12 \mathrm{C}, 8 \mathrm{C}, 6 \mathrm{C}$ or $4 \mathrm{C}$ ligands in solution-phase prior to film fabrication. c, Normalized TA spectra of the QD films with different ligands at 1 ps pump-probe time delay. Gradual red-shifts of the peaks were observed with shorter ligands. The fs-TAM data was taken near the corresponding positive $\Delta T / T$ photobleaching peaks with a bandwidth of $10 \mathrm{~nm}$ (shaded area). d, Representative fsbandwidth of $10 \mathrm{~nm}$ was achieved by using a bandpass filter $(730 \mathrm{~nm})$ in the imaging path. e, 
276 The corresponding exciton distribution images retrieved by fits with isotropic two-

277 dimensional Gaussian functions. The $\sigma$ values represent the width of exciton distribution at 278 the corresponding time delay. Clear expansion of exciton distribution over time can be 279 observed. Scale bars, $500 \mathrm{~nm}$.

280

281

282

283

284

285

286 

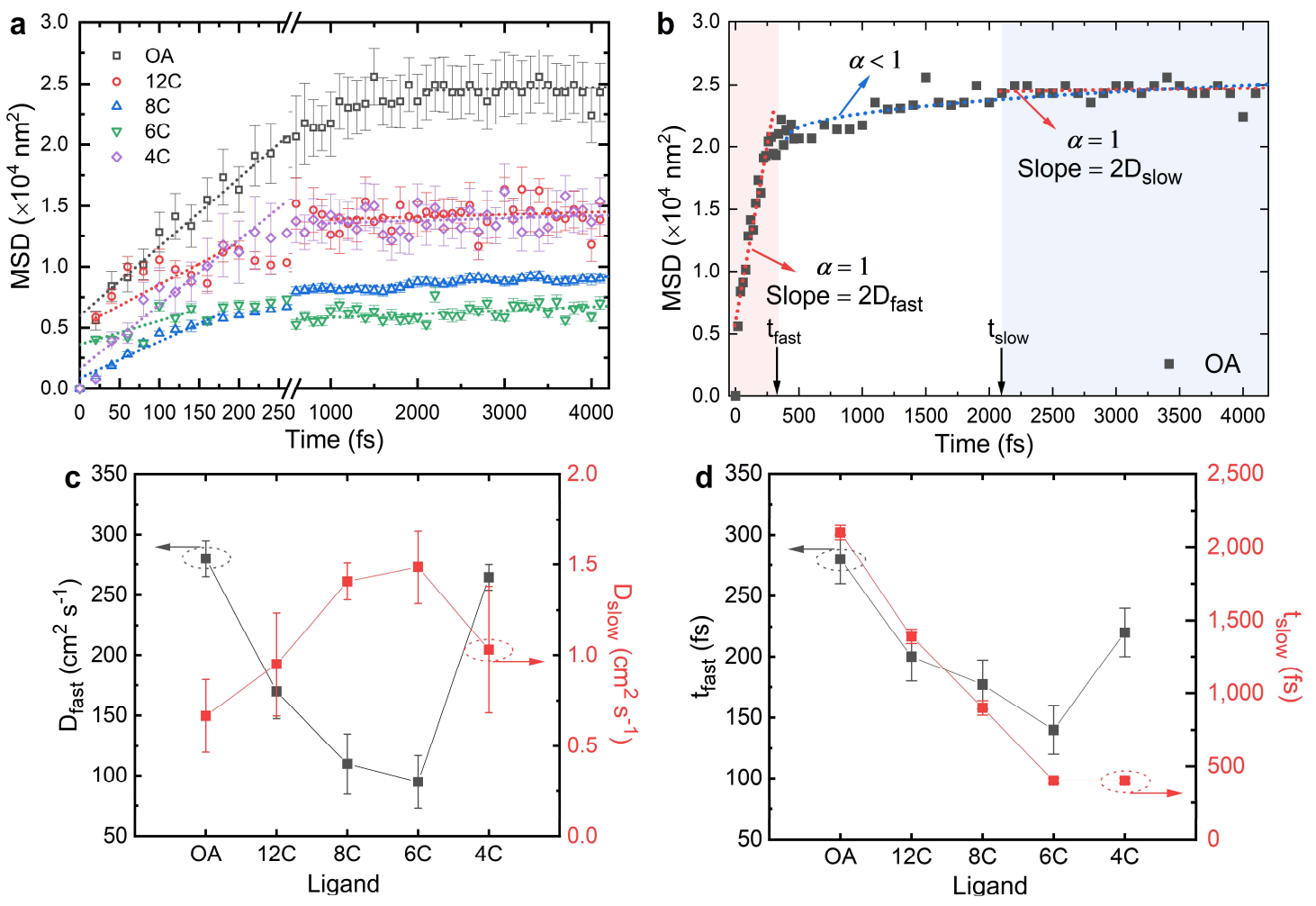

Fig. 2 Quantitative fs-TAM measurement results of the series of QD thin films. a, Time

evolution of the MSD $=\sigma_{t}^{2}-\sigma_{0}^{2}$ profile, while $\sigma_{0}^{2}$ represents the width of the spatial exciton

distribution near zero pump-probe delay. The dotted lines are the fits within the respective

time range based on the power law of $\mathrm{MSD}=2 D t^{\alpha}$, where $\alpha=1$. $\mathbf{b}$, The fitted curves and

resultants parameters extracted for a representative QD film, OA as an example. The MSD

profile within the time range of $0<t<t_{\text {fast }}$ and that of $t>t_{\text {slow }}$ can be well described by the

diffusive motion such that $\alpha=1$ from the power law equation (red dotted line), where the diffusivities of $D_{\text {fast }}$ and $D_{\text {slow }}$ can be extracted from the slope of the corresponding fitted lines. The MSD profile after $t_{\text {fast }}$ can also be described by a subdiffusive motion (blue dotted line, $\alpha<1$ ), while conclusions from the two fitting methods are consistent (Supplementary Discussion 2). c, The extracted diffusivities of the initial fast transport regime $\left(D_{\text {fast }}\right)$ and the slow regime $\left(D_{\text {slow }}\right)$ of the series of QD films. The two transport regimes show opposite 
300

301

302

303

304

305

306

307

308

309

310

311

312

313

314 

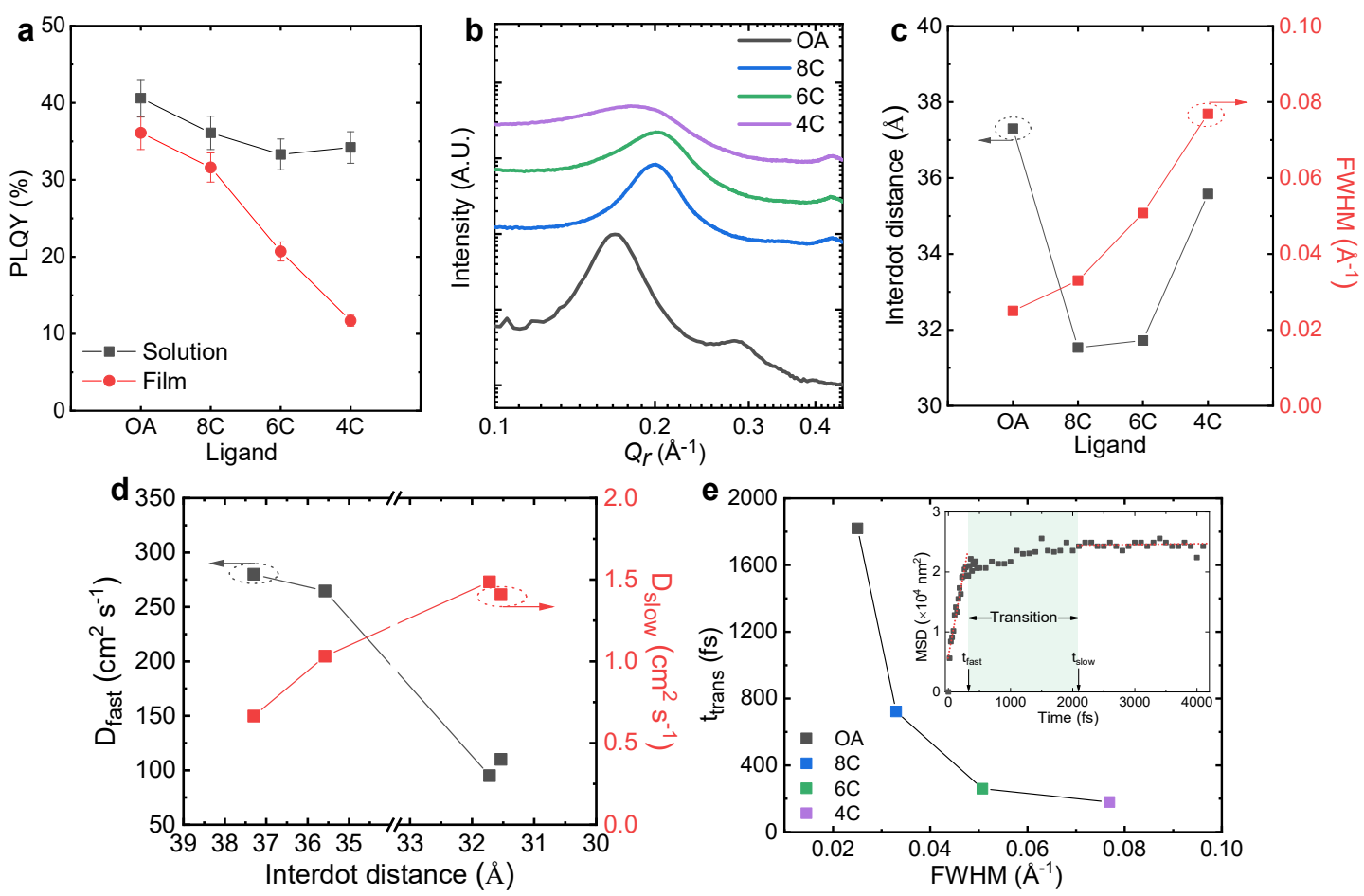

Fig. 3 Quality and structural information of the QDs and films with different ligands

and correlation to the fs-TAM results. a, PLQY of the QDs in both solution- and solid-

state with different ligands. A more significant quenching in the PLQY can be observed from

films with shorter ligands. No chemical or thermal treatment was applied to the films,

indicating that the quenching of PLQY arises from improved QD coupling due to closer

interdot distance. b, Radially integrated GISAXS intensities of QD solids (normalized).

Lower $Q_{r}$ value of the $1^{\text {st }}$ order scattering peaks indicates larger average separation between the QDs, while the peak width indicates discrepancy of the separation. c, The fitted interdot distance and FWHM of the $1^{\text {st }}$ order scattering peaks from $\mathbf{b}$. We ascribe the FWHM to a relative deviation in the core-to-core spacing of the QDs and hence level of packing disorder in the samples. d, Correlation between the actual interdot distance (core-to-core) with the diffusivities of the fast and slow regimes. The $D_{\text {fast }}$ decreases with reducing interdot distance while $D_{\text {slow }}$ clearly shows an opposite trend. e, Correlation between the transition time $\left(t_{\text {trans }}=\right.$ $t_{\text {slow }}-t_{\text {fast }}$, inset) with the FWHM (level of packing disorder) of the $1^{\text {st }}$ order scattering peaks 
330 in b. The decreasing $t_{\text {trans }}$ with increasing FWHM indicates higher level of disorder leads to

331 faster transition from the fast to the slow regime.

332

333

334

335

336

337

338

339

340

341

342

343

344

345 

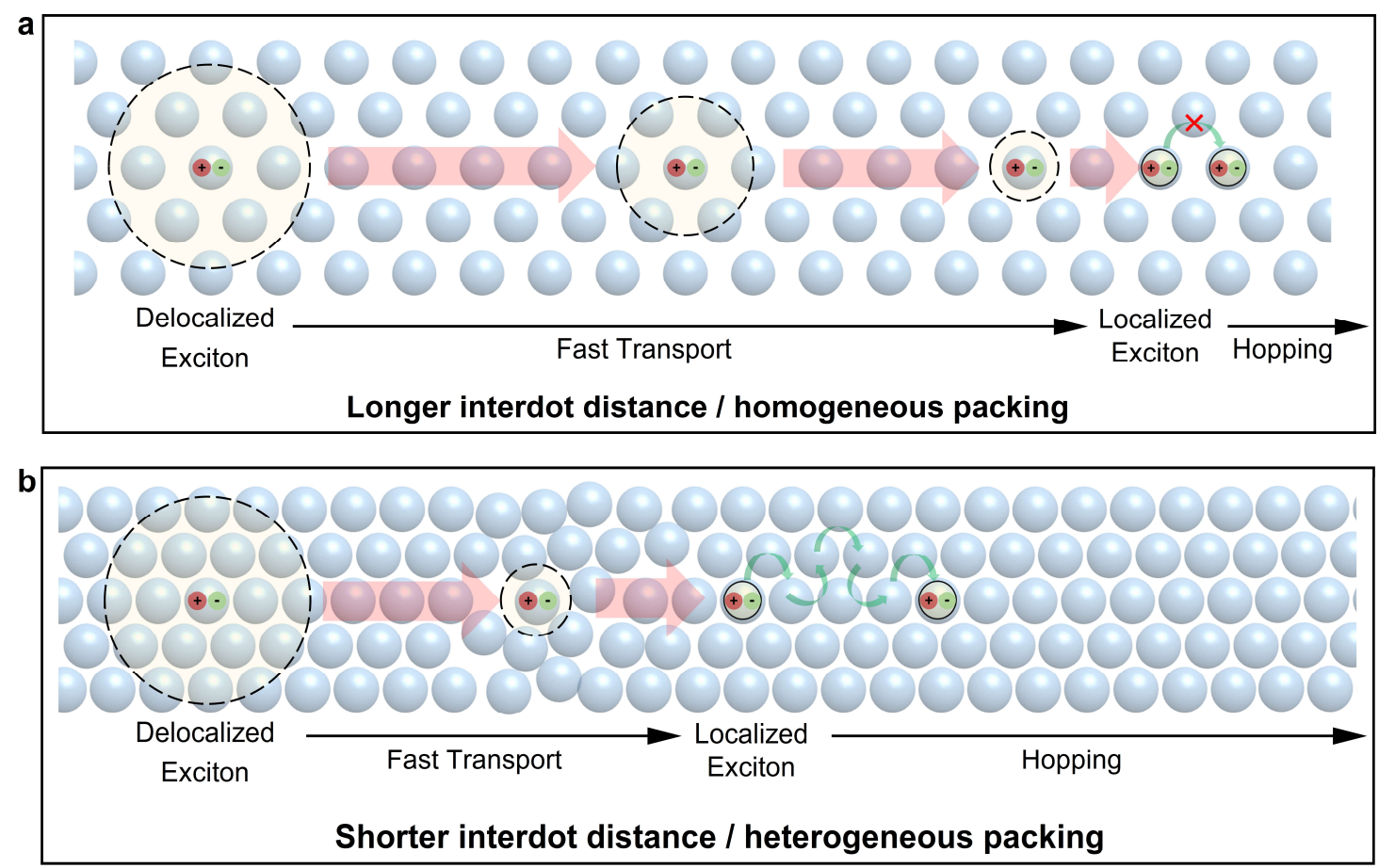

347 Fig. 4 Schematic of early time exciton transport in QD solids. a, The case of QD solids

348 with relatively long (but still closely-packed) interdot distance and/or homogeneous packing.

349 The photogenerated exciton initially delocalizes over multiple QDs and diffuses through the

350 QD solid rapidly. The low QD packing density and lack of disorder allows the transport to be

351 faster and sustained longer. As disorders still exist the exciton would eventually be localized,

352 and after the exciton hopping process is inefficient due to the long interdot distance. $\mathbf{b}$, The

353 case of QD solids with shorter interdot distance and/or heterogeneous packing. Due to high

354 QD packing density and the existence of packing disorder, the delocalized exciton diffuses

355 relatively slowly and can be localized much earlier. However, after localization the exciton

356 can keep moving among QDs through hopping due to the shorter interdot distance. 
361

362

363

364

365

366

367

368

369

370

371

372

373

374

375

376

377

378

379

380

381

382

383

384

385

386

387

388

389

390

391

392

393

394

395

396

397

398

399

400

401

402

403

404

405

406
1. Kagan, C. R., Lifshitz, E., Sargent, E. H. \& Talapin, D. V. Building devices from colloidal quantum dots. Science 353, aac5523. (2016)

2. Kagan, C. R. \& Murray, C. B. Charge transport in strongly coupled quantum dot solids. Nat. Nanotechnol. 10, 1013-1026. (2015)

3. Lee, J. S., Kovalenko, M. V., Huang, J., Chung, D. S. \& Talapin, D. V. Band-like transport, high electron mobility and high photoconductivity in all-inorganic nanocrystal arrays. Nat. Nanotechnol. 6, 348-352. (2011)

4. Sun, L., Choi, J. J., Stachnik, D., Bartnik, A. C., Hyun, B. R., Malliaras, G. G., et al. Bright infrared quantum-dot light-emitting diodes through inter-dot spacing control. Nat. Nanotechnol. 7, 369-373. (2012)

5. Liu, M., Voznyy, O., Sabatini, R., Garcia de Arquer, F. P., Munir, R., Balawi, A. H., et al. Hybrid organic-inorganic inks flatten the energy landscape in colloidal quantum dot solids. Nat. Mater. 16, 258-263. (2017)

6. Chuang, C. H., Brown, P. R., Bulovic, V. \& Bawendi, M. G. Improved performance and stability in quantum dot solar cells through band alignment engineering. Nat. Mater. 13, 796-801. (2014)

7. Akselrod, G. M., Prins, F., Poulikakos, L. V., Lee, E. M., Weidman, M. C., Mork, A. J., et al. Subdiffusive exciton transport in quantum dot solids. Nano Lett. 14, 35563562. (2014)

8. Yoon, S. J., Guo, Z., Dos Santos Claro, P. C., Shevchenko, E. V. \& Huang, L. Direct imaging of long-range exciton transport in quantum dot superlattices by ultrafast microscopy. ACS Nano 10, 7208-7215. (2016)

9. Lan, X., Chen, M., Hudson, M. H., Kamysbayev, V., Wang, Y., Guyot-Sionnest, P., et al. Quantum dot solids showing state-resolved band-like transport. Nat. Mater. 19, 323-329. (2020)

10. Crisp, R. W., Schrauben, J. N., Beard, M. C., Luther, J. M. \& Johnson, J. C. Coherent exciton delocalization in strongly coupled quantum dot arrays. Nano Lett. 13, 48624869. (2013)

11. Choi, J. H., Fafarman, A. T., Oh, S. J., Ko, D. K., Kim, D. K., Diroll, B. T., et al. Bandlike transport in strongly coupled and doped quantum dot solids: a route to highperformance thin-film electronics. Nano Lett. 12, 2631-2638. (2012)

12. Akselrod, G. M., Deotare, P. B., Thompson, N. J., Lee, J., Tisdale, W. A., Baldo, M. A., et al. Visualization of exciton transport in ordered and disordered molecular solids. Nat. Commun. 5, 3646. (2014)

13. Zhu, T., Wan, Y., Guo, Z., Johnson, J. \& Huang, L. Two birds with one stone: Tailoring singlet fission for both triplet yield and exciton diffusion length. $A d v$. Mater. 28, 7539-7547. (2016)

14. Ginsberg, N. S. \& Tisdale, W. A. Spatially resolved photogenerated exciton and charge transport in emerging semiconductors. Annu. Rev. Phys. Chem. 71, 1-30. (2020)

15. Sung, J., Schnedermann, C., Ni, L., Sadhanala, A., Chen, R. Y. S., Cho, C., et al. Long-range ballistic propagation of carriers in methylammonium lead iodide perovskite thin films. Nat. Phys. 16, 171-176. (2019)

16. Guo, Z., Manser, J. S., Wan, Y., Kamat, P. V. \& Huang, L. Spatial and temporal imaging of long-range charge transport in perovskite thin films by ultrafast microscopy. Nat. Commun. 6, 7471. (2015) 
17. Choi, J. J., Luria, J., Hyun, B. R., Bartnik, A. C., Sun, L., Lim, Y. F., et al. Photogenerated exciton dissociation in highly coupled lead salt nanocrystal assemblies. Nano Lett. 10, 1805-1811. (2010)

18. Schnedermann, C., Sung, J., Pandya, R., Verma, S. D., Chen, R. Y. S., Gauriot, N., et $a l$. Ultrafast tracking of exciton and charge carrier transport in optoelectronic materials on the nanometer scale. J. Phys. Chem. Lett. 10, 6727-6733. (2019)

19. Zhang, J., Tolentino, J., Smith, E. R., Zhang, J., Beard, M. C., Nozik, A. J., et al. Carrier transport in $\mathrm{PbS}$ and $\mathrm{PbSe} \mathrm{QD}$ films measured by photoluminescence quenching. J. Phys. Chem. C 118, 16228-16235. (2014)

20. Contreras-Pulido, L. D. \& Bruderer, M. Coherent and incoherent charge transport in linear triple quantum dots. J. Phys. Condens Matter. 29, 185301. (2017)

21. Barford, W. \& Duffy, C. D. P. Role of quantum coherence and energetic disorder in exciton transport in polymer films. Phys. Rev. B 74, 075207. (2006)

22. Cohen, E., Gdor, I., Romero, E., Yochelis, S., van Grondelle, R. \& Paltiel, Y. Achieving exciton delocalization in quantum dot aggregates using organic linker molecules. J. Phys. Chem. Lett. 8, 1014-1018. (2017)

23. Lee, E. M. Y., Tisdale, W. A. \& Willard, A. P. Perspective: Nonequilibrium dynamics of localized and delocalized excitons in colloidal quantum dot solids. J. Vac. Sci. Technol. A 36, 068501. (2018)

24. Sharma, A., Zhang, L., Tollerud, J. O., Dong, M., Zhu, Y., Halbich, R., et al. Supertransport of excitons in atomically thin organic semiconductors at the 2D quantum limit. Light Sci. Appl. 9, 116. (2020)

25. Fratini, S., Nikolka, M., Salleo, A., Schweicher, G. \& Sirringhaus, H. Charge transport in high-mobility conjugated polymers and molecular semiconductors. Nat. Mater. 19, 491-502. (2020)

26. Schweicher, G., D'Avino, G., Ruggiero, M. T., Harkin, D. J., Broch, K., Venkateshvaran, D., et al. Chasing the "killer" phonon mode for the rational design of low-disorder, high-mobility molecular semiconductors. Adv. Mater. 31, e1902407. (2019)

\section{Acknowledgement}

This work has received funding from the European Research Council (ERC) under the European Union's Horizon 2020 research and innovation programme (grant agreement number 758826). Z. Z. acknowledges funding from the European Union's Horizon 2020

research and innovation programme under the Marie Skłodowska-Curie Actions grant (No.

842271 - TRITON project). D. T. W. T., M. P. W., A. J. R. and R. A. L. J. acknowledge support from the Engineering and Physical Sciences Research Council (UK) via grant EP/P027814/1. We thank Dr. David Paleček for the assistance with the TA spectroscopy 
445 measurements, and Dr. Christoph Schnedermann for the useful discussion on transport

446 dynamics.

447

448 Author contributions

449 Z.Z., J.S. and A.R. conceived the project, performed the sample synthesis and fabrication, fs-

450 TAM and other optical measurements, and wrote the paper. D.T.W.T., M.P.W., A.J.R. and

451 R.A.L.J. supported and performed the GISAXS measurements and analysis. SY.H and M.L.

452 provided input into the sample preparation. J.X. conducted electron microscopy

453 measurements. S.D. contributed to PLQY measurement setups. SJ.H. provided input to the

454 design of experiments and discussion of results.

455

456 Additional information

457 Supplementary information is available in the online version of the paper. Correspondence

458 and requests for materials should be addressed to A.R.

459

460 Competing financial interests

461 The authors declare no competing financial interests.

462

463

464 


\section{Figures}
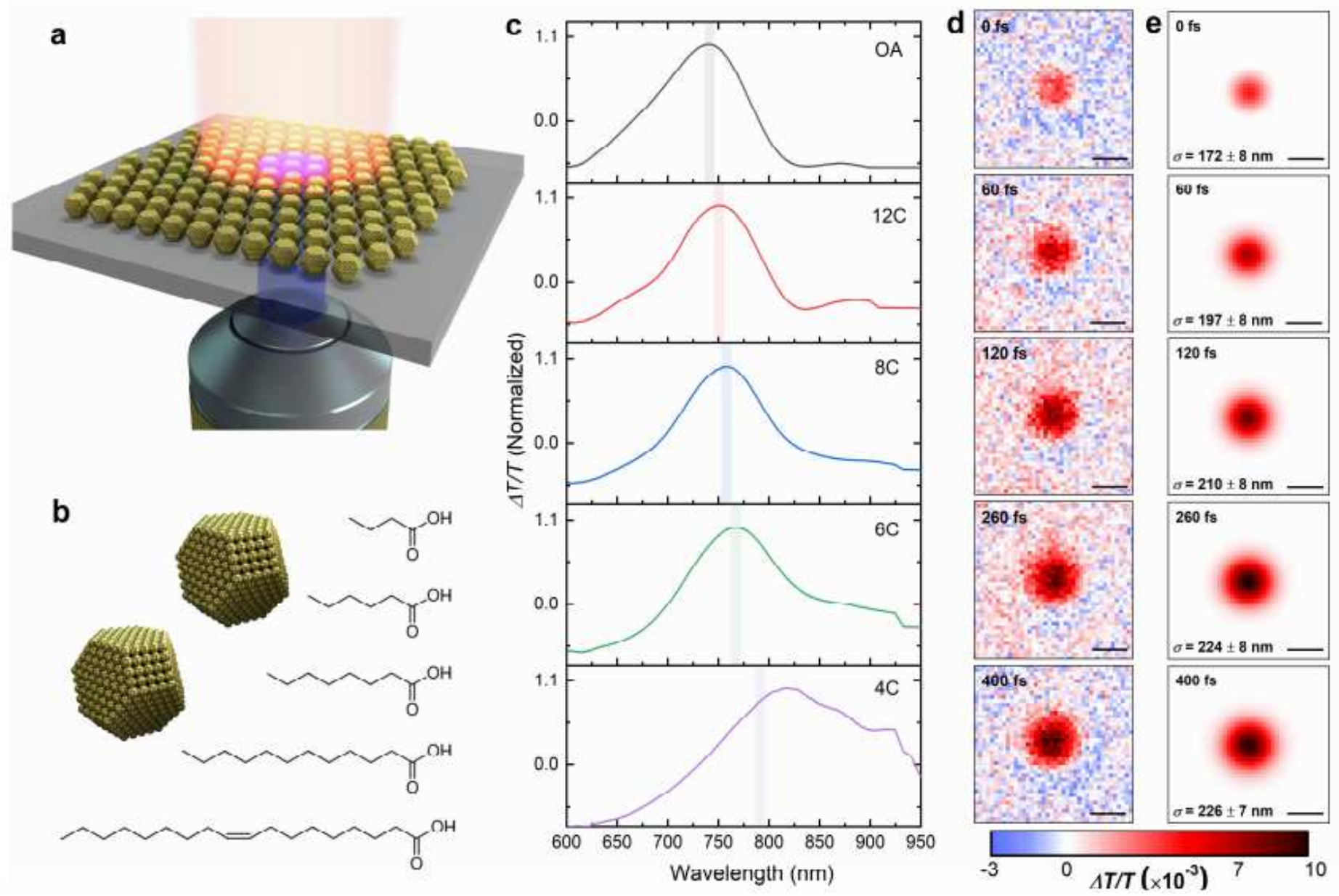

\section{Figure 1}

The fs-TAM measurement of QD thin films with different capping ligands as discussed in this work. a, Schematic representation of the fs-TAM measurement. A near diffraction-limited ( $\sigma$ of $107 \pm 7.5 \mathrm{~nm}$ ) and transform-limited ( $9.2 \mathrm{fs}, 580 \mathrm{~nm}$ ) pump beam is delivered onto the QD film (blue beam), together with a counter-propagating loosely focused $(\sigma$ of $6.4 \mu \mathrm{m}$ ) and transform-limited (6.8 fs) probe pulse (orange beam). Note that the actual samples are thicker films containing multiple layers of QDs with total thickness about $200 \mathrm{~nm}$. b. The interdot distance of the films were modified by the selection of surface ligands. The ligand exchange was performed to replace the original OA ligands to $12 \mathrm{C}, 8 \mathrm{C}, 6 \mathrm{C}$ or $4 \mathrm{C}$ ligands in solution-phase prior to film fabrication. c, Normalized TA spectra of the QD films with different ligands at 1 ps pump-probe time delay. Gradual red-shifts of the peaks were observed with shorter ligands. The fs-TAM data was taken near the corresponding positive $\triangle T / T$ photobleaching peaks with a bandwidth of $10 \mathrm{~nm}$ (shaded area). d, Representative fs- TAM images of OA at 0, 60, 120, 260 and $400 \mathrm{fs}$ pump-probe delay, respectively. A spectral bandwidth of $10 \mathrm{~nm}$ was achieved by using a bandpass filter $(730 \mathrm{~nm})$ in the imaging path. e, The corresponding exciton distribution images retrieved by fits with isotropic two- dimensional Gaussian functions. The $\sigma$ values represent the width of exciton distribution at 
the corresponding time delay. Clear expansion of exciton distribution over time can be observed. Scale bars, $500 \mathrm{~nm}$.
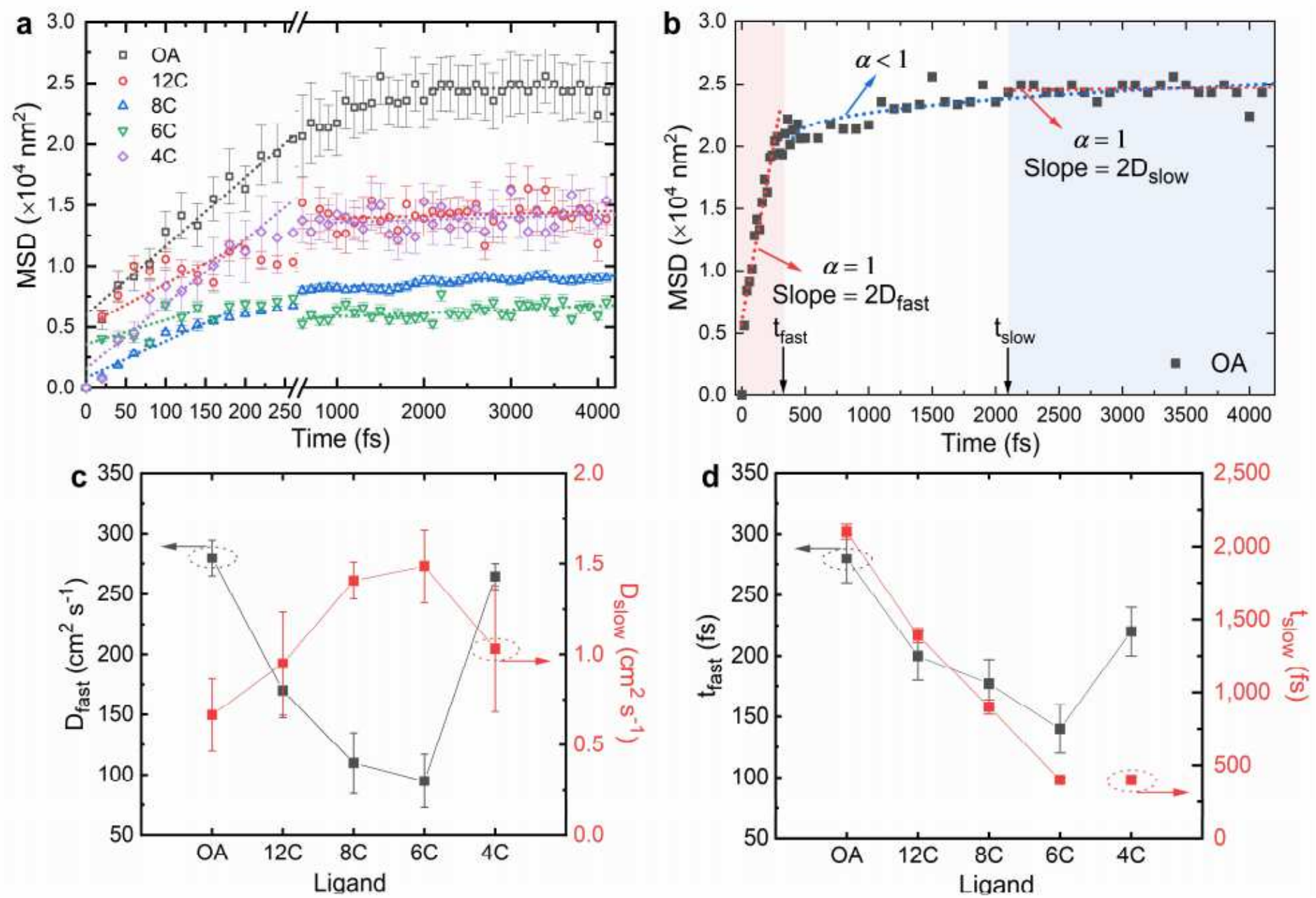

Figure 2

Quantitative fs-TAM measurement results of the series of QD thin films. a, Time evolution of the MSD = ot2 $-\sigma 2$ profile, while $\sigma 2$ represents the width of the spatial exciton distribution near zero pump-probe delay. The dotted lines are the fits within the respective time range based on the power law of MSD = $2 D t a$, where $a=1 . b$, The fitted curves and resultants parameters extracted for a representative QD film, $\mathrm{OA}$ as an example. The MSD profile within the time range of $0<\mathrm{t}<\mathrm{tfast}$ and that of $\mathrm{t}>\mathrm{tsl}$. described by the diffusive motion such that $\alpha=1$ from the power law equation (red dotted line), where the diffusivities of Dfast and Dslow can be extracted from the slope of the corresponding fitted lines. The MSD profile after tfast can also be described by a subdiffusive motion (blue dotted line, $a<1$ ), while conclusions from the two fitting methods are consistent (Supplementary Discussion 2). c, The extracted diffusivities of the initial fast transport regime (Dfast) and the slow regime (Dslow) of the series of QD films. The two transport regimes show opposite response to the variation of ligands. $d$, The estimated time duration of the initial fast stage (tfast) and the time taken (from $0 \mathrm{fs}$ ) for the excitons to enter the slow transport regime (tslow). 

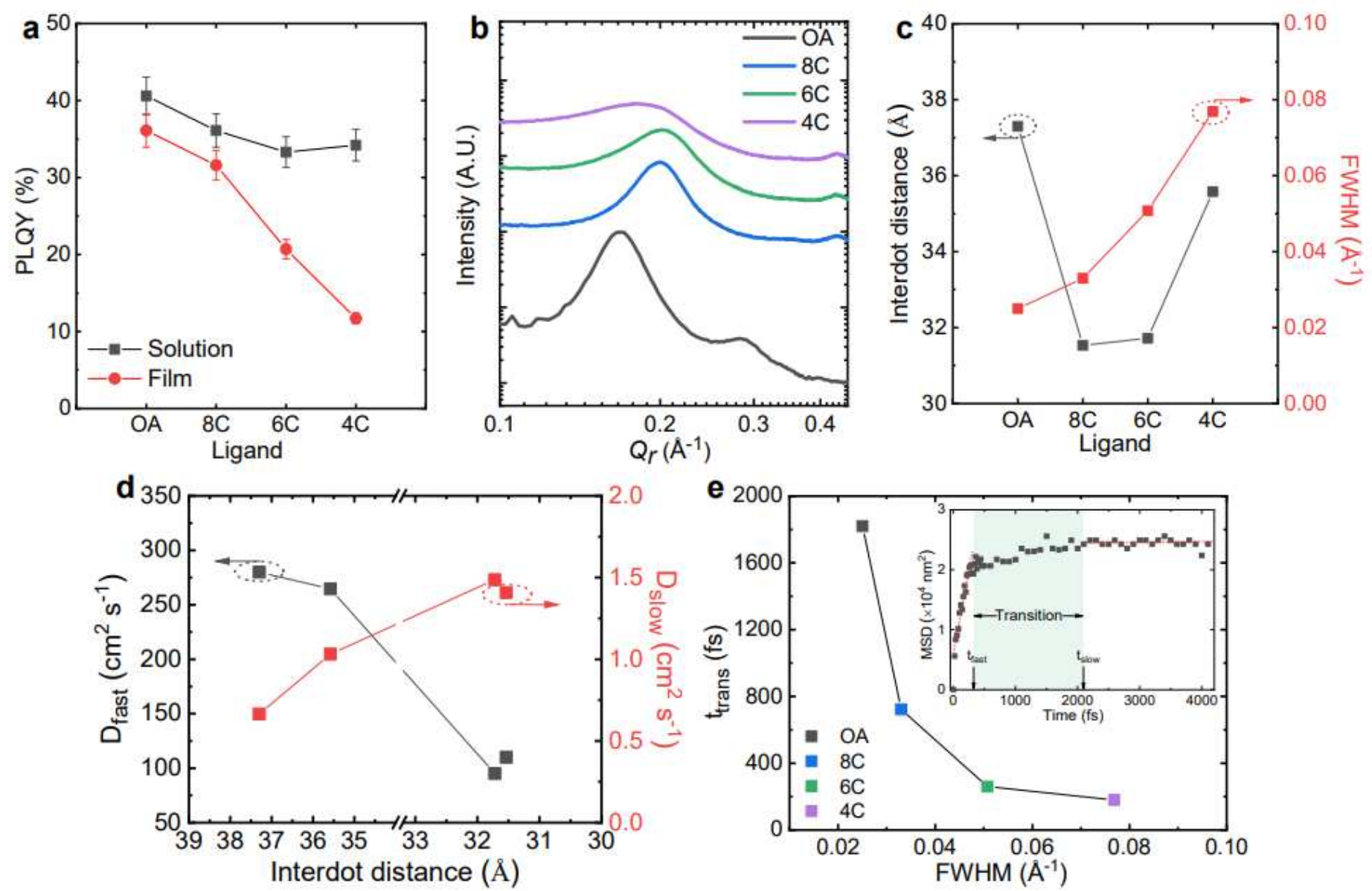

\section{Figure 3}

Quality and structural information of the QDs and films with different ligands and correlation to the fsTAM results. a, PLQY of the QDs in both solution- and solid- state with different ligands. A more significant quenching in the PLQY can be observed from films with shorter ligands. No chemical or thermal treatment was applied to the films, indicating that the quenching of PLQY arises from improved QD coupling due to closer interdot distance. b, Radially integrated GISAXS intensities of QD solids (normalized). Lower Qr value of the 1st order scattering peaks indicates larger average separation between the QDs, while the peak width indicates discrepancy of the separation. c, The fitted interdot distance and FWHM of the 1 st order scattering peaks from $\mathrm{b}$. We ascribe the FWHM to a relative deviation in the core-to-core spacing of the QDs and hence level of packing disorder in the samples. $d$, Correlation between the actual interdot distance (core-to-core) with the diffusivities of the fast and slow regimes. The Dfast decreases with reducing interdot distance while Dslow clearly shows an opposite trend. e, Correlation between the transition time (ttrans = tslow - tfast, inset) with the FWHM (level of packing disorder) of the 1 st order scattering peaks in $\mathrm{b}$. The decreasing ttrans with increasing FWHM indicates higher level of disorder leads to faster transition from the fast to the slow regime. 

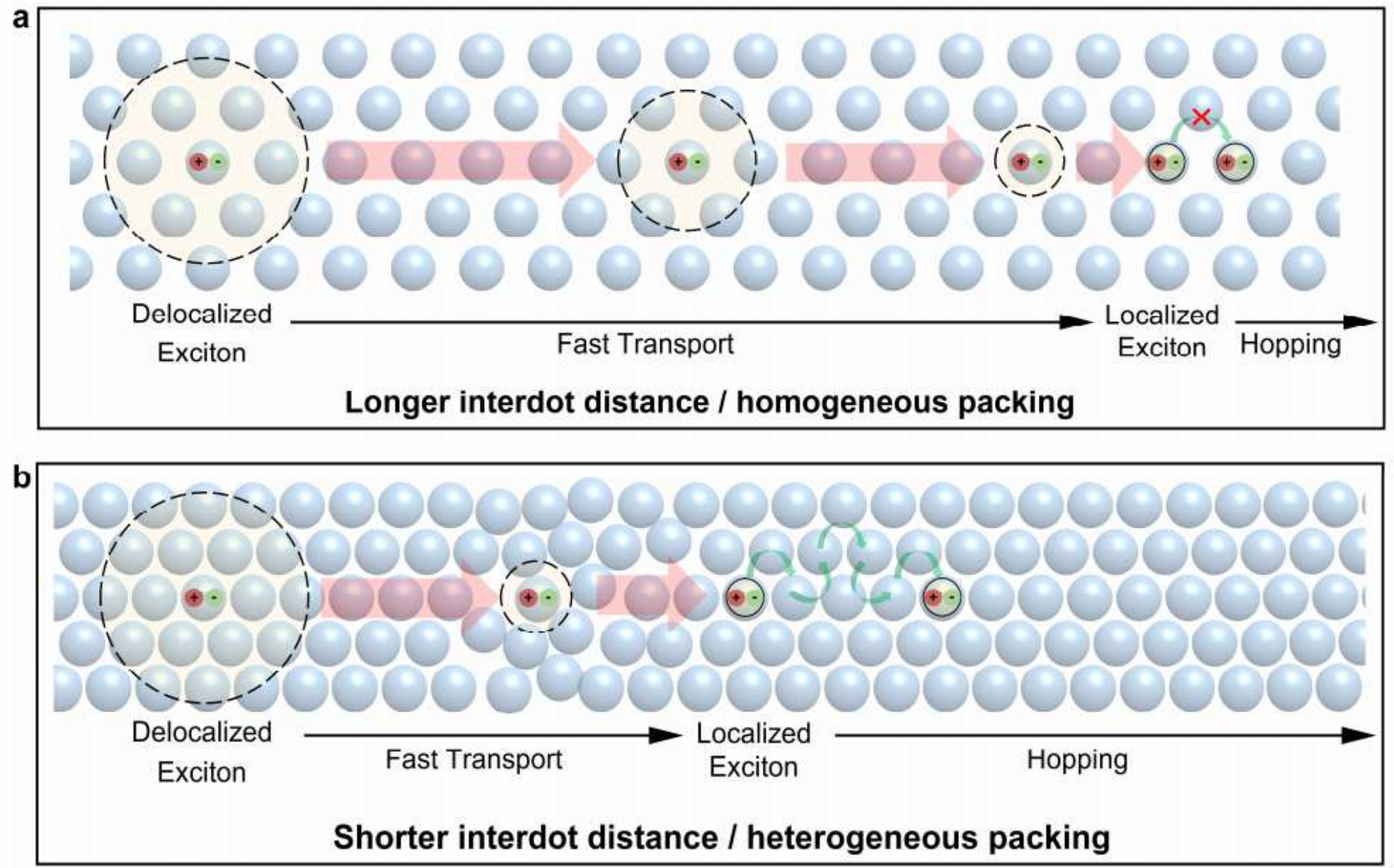

Figure 4

Schematic of early time exciton transport in QD solids. a, The case of QD solids with relatively long (but still closely-packed) interdot distance and/or homogeneous packing. The photogenerated exciton initially delocalizes over multiple QDs and diffuses through the QD solid rapidly. The low QD packing density and lack of disorder allows the transport to be faster and sustained longer. As disorders still exist the exciton would eventually be localized, and after the exciton hopping process is inefficient due to the long interdot distance. b, The case of QD solids with shorter interdot distance and/or heterogeneous packing. Due to high QD packing density and the existence of packing disorder, the delocalized exciton diffuses relatively slowly and can be localized much earlier. However, after localization the exciton can keep moving among QDs through hopping due to the shorter interdot distance.

\section{Supplementary Files}

This is a list of supplementary files associated with this preprint. Click to download.

- SupplementaryInformationQDultrafastsubmission.pdf 